\title{
Interrupted time-series analysis: studying trends in neurosurgery
}

\author{
Ricky H. Wong, MD, ${ }^{1}$ Fabrice Smieliauskas, PhD, ${ }^{2}$ I-Wen Pan, PhD, ${ }^{3}$ and Sandi K. Lam, MD, MBA ${ }^{3}$ \\ ${ }^{1}$ Department of Neurosurgery, University of South Florida, Tampa, Florida; ${ }^{2}$ Department of Health Sciences, University of \\ Chicago, Illinois; and '3epartment of Neurosurgery, Baylor College of Medicine, Texas Children's Hospital, Houston, Texas
}

\begin{abstract}
OBJECT Neurosurgery studies traditionally have evaluated the effects of interventions on health care outcomes by studying overall changes in measured outcomes over time. Yet, this type of linear analysis is limited due to lack of consideration of the trend's effects both pre- and postintervention and the potential for confounding influences. The aim of this study was to illustrate interrupted time-series analysis (ITSA) as applied to an example in the neurosurgical literature and highlight ITSA's potential for future applications.
\end{abstract}

METHODS The methods used in previous neurosurgical studies were analyzed and then compared with the methodology of ITSA.

RESULTS The ITSA method was identified in the neurosurgical literature as an important technique for isolating the effect of an intervention (such as a policy change or a quality and safety initiative) on a health outcome independent of other factors driving trends in the outcome. The authors determined that ITSA allows for analysis of the intervention's immediate impact on outcome level and on subsequent trends and enables a more careful measure of the causal effects of interventions on health care outcomes.

CONCLUSIONS ITSA represents a significant improvement over traditional observational study designs in quantifying the impact of an intervention. ITSA is a useful statistical procedure to understand, consider, and implement as the field of neurosurgery evolves in sophistication in big-data analytics, economics, and health services research.

http://thejns.org/doi/abs/10.3171/2015.9.FOCUS15374

KEY WORDS interrupted time series; kyphoplasty; methodology; segmented time series; trends

$\mathrm{T}$ HE cost of health care in the US is rising, and health care in the US has come under increasing scrutiny for purportedly providing costly care that yields equivocal benefits. This increased attention has led to a growing focus on improving the quality and cost-effectiveness of care. To that end, the number of publications pertaining to cost reduction and quality improvement has increased dramatically. A variety of methods have been developed in statistics and econometrics for estimating the causal effects of interventions on health care outcomes. ${ }^{12}$

The surgical literature typically evaluates policy changes by studying overall changes in outcomes over time, with little attempt to estimate whether and how much of the change truly can be attributed to the intervention. The perils of this approach are best illustrated by considering the increase in spinal fusion procedures over the past de- cade. This increase continued despite the introduction of alternatives to fusion for patients with lumbar degenerative disc disease, including disc arthroplasty, annuloplasty, and posterior dynamic stabilization. Using the method of making inferences from simple changes in fusion rates over the decade would suggest that introducing these procedures led to more fusions. More likely, some patients underwent these procedures rather than undergoing fusions, so the procedures actually reduced the number of fusions. But, simultaneously, an increase in the diagnosis of back pain and greater awareness of surgical options led to a growing number of fusions regardless.

An interrupted time-series analysis (ITSA) is a quasiexperimental method for isolating the effect of an intervention on a health outcome independent of other factors driving trends in the outcome. ${ }^{23}$ ITSA generates an esti-

ABBREVIATIONS AAOS = American Academy of Orthopaedic Surgeons; ACS NSQIP = American College of Surgeons National Surgical Quality Improvement Program; ITSA = interrupted time-series analysis; $\mathrm{N}^{2} \mathrm{QOD}=$ National Neurosurgery Quality and Outcomes Database; VAP = vertebral augmentation procedure; VCF = vertebral compression fracture. 
mate of the causal effect by comparing the actual outcome some time after the intervention transpires with what the outcome would have been, as determined by the preexisting level and trend path of the outcome. Although this counterfactual, hypothetical outcome had never occurred, ITSA projects what that outcome would have been by extrapolating the trend line of the outcome from the period before the intervention, as though the intervention had never occurred. When an intervention of interest occurs during that time period, its effect is captured as a change in the level, a change in trend over time, or both. The level in an ITSA denotes the value of the outcome measure at a particular time interval, while the trend represents the rate of change (or slope) of the level. Using segmented regression analysis, regression lines can be assigned to pre- and postintervention time segments of the outcome data. This enables analysis of the intervention's immediate impact on outcome level and impact on a subsequent trend that provides a more nuanced measure of the intervention's impact. It also allows construction of confidence intervals for the overall change; thus, inferences can be drawn to determine whether the overall change is statistically significant.

In this study, we examined the methods used in previous neurosurgical studies and compared them with the methodology of ITSA highlighted here. Our goals were to illustrate ITSA as applied to an example in the neurosurgical literature and highlight its potential for future applications.

\section{Methods}

In 2009, 2 randomized controlled trials investigating the efficacy of vertebroplasty versus that of sham surgery for patients with osteoporotic vertebral compression fractures (VCFs) were published. ${ }^{5,13}$ The studies found no differences in pain or disability between the 2 groups. Subsequently, the American Academy of Orthopaedic Surgeons (AAOS) advised against the use of vertebroplasty in patients with osteoporotic VCFs, stating that evidence supporting its use is "limited." Three recent publications have attempted to determine the impact of these events on the national use of vertebral augmentation procedures (VAPs). ${ }^{9,15,22}$ We examined the methods and results performed in these studies in comparison with the ITSA method.

A brief outline of ITSA is given here with definitions and parameters of interest. A time series is a sequence of values taken at regular intervals over time, while segments in a time series are divided up portions at change points. The specific points in time when values of a time series exhibit change from previously established patterns are termed "change points," typically linked to real-world events such as policy change or other interventions. With each segment of a time series, a level and a trend occur. The level is the value at the beginning of the time interval, which is essentially the y-intercept. The trend is the rate of change, which is the slope of the segment. Building a final model in segmented regression analysis needs to take into account the level and trend, and changes thereof, in the pre- and postintervention time frames. Depending on the data collected, outcome measures can typically be expressed as averages, proportions, or rates. The recommended minimum number of data points is 12 in each of the pre- and postintervention time periods. At each data point of the time series, a minimum of 100 observations is recommended to have an acceptable level of variability of the estimate at any particular time point. After running the full regression model, stepwise backward elimination with a predetermined significance level for predictive factors as well as a priori selected important variables produces the parsimonious model. The fit of the final model should also be assessed. Overall, the equation is represented as follows:

$$
\begin{aligned}
& Y_{t}=\beta_{0}(\text { constant })+\beta_{1} * \text { time }_{t}+\beta_{2} * \text { intervention }_{t}+ \\
& \beta_{3} * \text { time after intervention } \\
& t
\end{aligned}
$$

where $Y_{t}$ represents rates, percentage, or utilization, such as number of surgeries in month " $t$ "; $\beta_{0}$ estimates baseline level of the outcome (such as mean number of surgeries per month); $\beta_{1}$ estimates change in the mean number of surgeries per month occurring in each month before the intervention, representing the baseline trend; $\beta_{2}$ estimates the level change in the mean number of surgeries per month immediately after the intervention (at the tail end of the preceding segment); $\beta_{3}$ estimates the change in the trend in mean number of surgeries after the intervention compared with the preintervention trend; time is a continuous variable, counting here by time in months; intervention $n_{\mathrm{t}}$ marks an indicator for time " $\mathrm{t}$ " occurring preintervention (intervention $=0$ ) or postintervention (intervention $=1)$; $e_{t}$ represents the error term at time " $t$ " for random variability not explained by the model; and $\beta_{1}+\beta_{3}$ gives the postintervention slope.

\section{Results}

Goz et al. analyzed data from the National Inpatient Sample database between 2005 and 2010. ${ }^{9}$ Using annual surgeries per 100,000 persons in their analysis, they found that kyphoplasty utilization increased from 27 procedures per 100,000 persons in 2005 to 33 procedures per 100,000 persons in 2007 , before dropping to 23 procedures per 100,000 persons in 2010 , whereas vertebroplasty utilization remained constant from 2005 to 2007 before briefly increasing and then decreasing in 2010. The authors concluded that evidence from the two aforementioned randomized controlled trials in 2009 had an effect on both vertebroplasty and kyphoplasty utilization.

Manchikanti et al. used data taken from the standard $5 \%$ national sample of the Centers for Medicare and Medicaid Services physician outpatient billing claims from 2001 to 2008 and the Physician/Supplier Procedure Summary Master File for 2009 and 2010. ${ }^{15}$ Their results and conclusions, overall, were similar to those of Goz et al. ${ }^{9}$

The present authors previously published an analysis on the impact of the trials on VAP using ITSA..$^{22}$ Using the Florida Hospital Inpatient Discharge and Ambulatory Surgery Center databases from 2005 to 2012, we captured a $100 \%$ sample of surgical spine procedures performed in hospitals, hospital surgical centers, and freestanding surgical centers in Florida. For the analysis, we allowed an interruption of the data in the third quarter of 2009, 
when the trials were published, and estimated the effects of the trials as of the fourth quarter of 2012. The regressions also accounted for seasonal fluctuations in vertebral augmentation rates and controlled for average patient age and percentage of procedures by sex, site of care, payer, and diagnosis. We used rates of spinal fusions and spinal disc procedures as controls for our analysis.

Both kyphoplasty and vertebroplasty rates declined following the publication of the randomized controlled trial results. While kyphoplasty exhibited an immediate effect, there was a lag of approximately 1 year before vertebroplasty declined. The simple change in rates of vertebroplasties and kyphoplasties from 2008 to 2012 was $-25 \%$ and $-9 \%$, respectively. Spinal fusion procedure and spinal disc procedure rates, which should have been unaffected by the publication of the vertebroplasty trials, did not decline in 2009 and were relatively unchanged over the period of 2009 to 2012.

The ITSA estimates of the effect of the trials demonstrated an overall decline in the vertebroplasty rate by $51.5 \%$ and the kyphoplasty rate by $40.0 \%$ as of the fourth quarter of 2012, because of the trials, and both results were statistically significant. For vertebroplasties, the change in the rate was driven by a time trend effect, whereas for kyphoplasties, both a level effect and a time trend effect were important contributors.

To evaluate for the possible role of changes in reimbursement, coverage policies for 6 of the largest private insurers and for the Centers for Medicare and Medicaid services were reviewed for the time period studied. There were no documented changes in coverage policy for these procedures during this time.

\section{Discussion}

Traditional observational studies that attempt to assess the impact of a practice or policy change use a mean representation of the preintervention data and compare that with a mean representation of the postintervention data. This analysis is limited by its lack of consideration for both the trends pre- and postintervention and the potential for confounding influences, among other weaknesses. These limitations make it more difficult to clearly attribute observed changes to a specific intervention.

Above, we illustrated how such limitations can compromise studies of procedure utilization trends. Recent studies $^{9,15}$ investigating the impact of randomized controlled trials on the use of VAP were able to suggest only that a decrease in the procedure numbers appeared to occur in 2009 and that this was likely to have been impacted by published trials.

The same is largely true for quality improvement measurement, provider profiling, and pay-for-performance methods currently being implemented for neurosurgeons and hospitals performing neurosurgery as part of health reform. The American College of Surgeons National Surgical Quality Improvement Program (ACS NSQIP) has developed advanced quality profiling techniques in surgery that have been adopted in neurosurgical research.,20,21 The ACS NSQIP has sophisticated risk adjustment for patient case mix,${ }^{6}$ but its provider report cards focus on cross-sec- tional quality comparisons of providers at 1 point in time ${ }^{14}$ and do not reflect quality improvements over time within institutions. A great majority of studies examining the impact of quality improvement initiatives on surgical quality rely on pre- and postintervention comparisons. ${ }^{10,11,14}$

By contrast, ITSA provides significantly more precise impact estimates than simple pre- and postintervention comparisons. In the VAP utilization rate example above, while both the simple changes and the ITSA estimates were negative for both VAPs, the magnitude of the declines was more than twice as large for the ITSA estimates ( $-51.5 \%$ vs $-25.0 \%$ for vertebroplasty; $-40.0 \%$ vs $-9.0 \%$ for kyphoplasty). ITSA captured more information, taking into account the fact that rates were on an upswing that was abruptly halted by the trials, which prevented further increases from occurring. Figure 1 intuitively illustrates how the ITSA estimates are obtained (regression controls for additional variables are not depicted here). In certain cases, as demonstrated in the hypothetical example of spinal fusion and fusion alternatives, ITSA can obtain changes of the opposite sign, unlike simple pre- and postintervention estimates.

Statistical inference can also be performed on ITSA estimates, which in this case turns out to be important, because one could question whether the simple 9\% decline in kyphoplasty was a genuine decline or simply random noise. Moreover, ITSA deconstructs changes into both level and trend change components. In our analysis of utilization rates of VAPs, we found that while both kyphoplasty and vertebroplasty rates declined after the trials, the decline in vertebroplasty rate was seen approximately 1 year later, coinciding more closely with the AAOS's statement on VAPs, and comprised only a trend change. These subtleties easily could be missed in pre- and postintervention studies.

Another significant benefit of ITSA is the ability to limit confounding factors that threaten the validity of effect estimates. Inherently, the design of an ITSA makes it difficult for significant confounding, since a confounder would need to occur at approximately the same time as the study intervention to produce an effect simultaneously. Using a control group can separate these differences even further. In the VAP analysis, we found that rates of spinal fusions and spinal disc procedures-control procedures that should be affected by any confounding influences common to spinal procedures-displayed no significant changes after the publication of the trials, even as VAP rates declined.

ITSA, by modeling risk-adjusted univariate time series, also offers a statistical framework for studying quality improvement impact for individual providers or for studying quality improvement impact when all providers are subject simultaneously to the same intervention, which has been used in select research on surgical quality.,3,16,18,19 When only some providers receive a quality improvement intervention, or receive it at different times, longitudinal data methods, such as fixed-effects regressions, can be used instead. ${ }^{8}$ With the development of the longitudinal National Neurosurgery Quality and Outcomes Database $\left(\mathrm{N}^{2} \mathrm{QOD}\right.$, www.neuropoint.org) and other outcomes data sets, the scope for application of these methods to analyze 


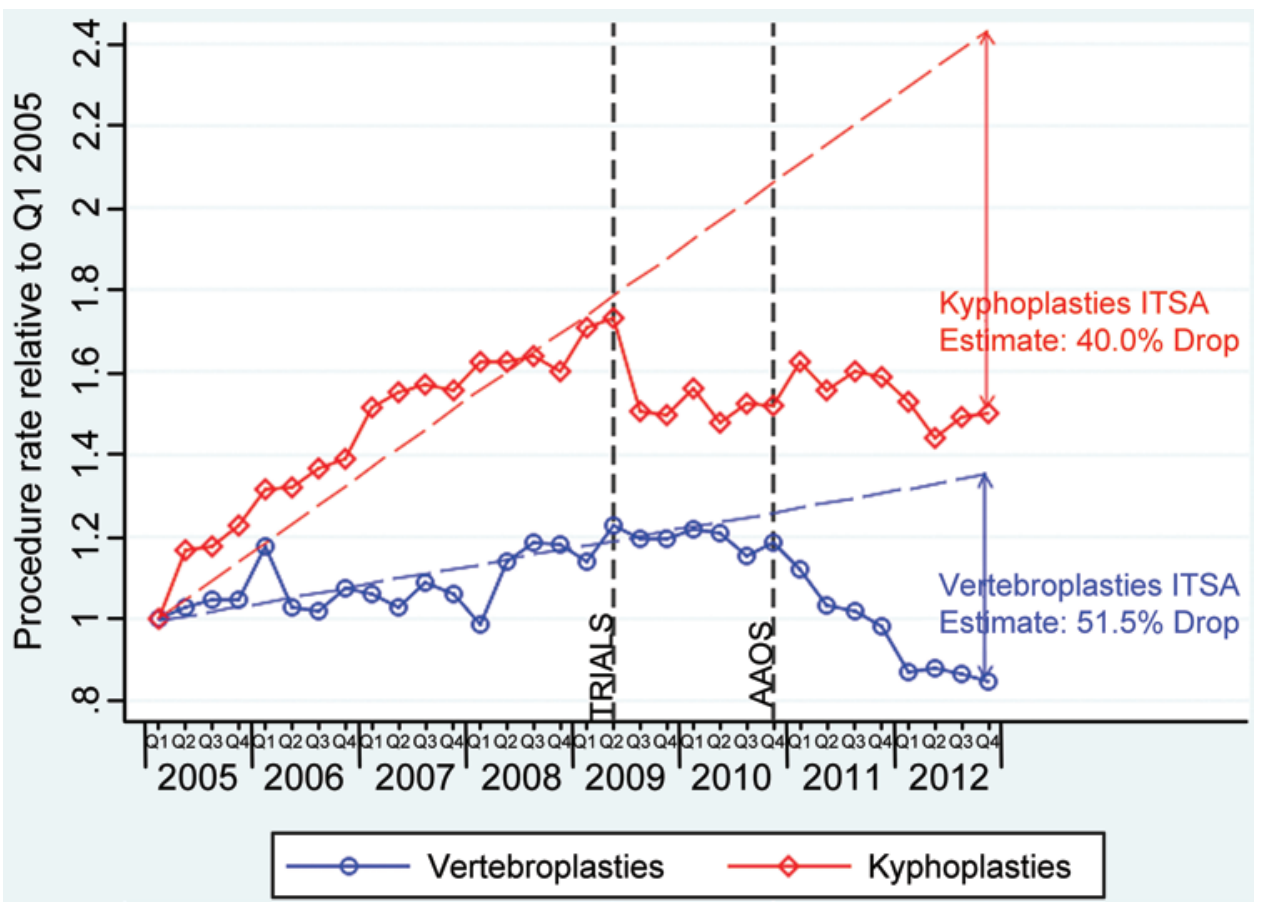

FIG. 1. Graph of common spine procedure rates per 100,000 adults. Procedure rates per 100,000 adults in the first quarter (Q1) of 2005 are as follows: vertebroplasties, 3.8; kyphoplasties, 8.1. All rates are seasonally adjusted. The "TRIALS" line indicates the quarter prior to the publication of the vertebroplasty trials. The "AAOS" line indicates the quarter prior to the release of the AAOS's statement on VAPs.

effects of neurosurgery quality improvement initiatives will only increase.

While it is beyond the scope and time frame of this paper, some relevant developments and trials warrant further study by ITSA in the near future. Results from clinical trials continually inform neurosurgical practice. For instance, A Randomized Trial of Unruptured Brain Arteriovenous Malformations (ARUBA) published in February 2014 in The Lancet brings new information to light regarding medical management versus interventional treatment for unruptured arteriovenous malformations. ${ }^{17}$ Additionally, the July 2014 New England Journal of Medicine publication on the results of a randomized trial of epidural glucocorticoid injections for spinal stenosis ${ }^{7}$ will be another interesting example. Over time, we need to explore and understand how these trials affect the rate of intervention and impact clinical practice in the US.

The major limitation of ITSA is the need to assume that outcomes would have followed a preexisting trend in the absence of the intervention being studied. Standard assumptions are that the trend is linear and would continue in perpetuity. Careful consideration of the preintervention data is needed to determine whether a linear or nonlinear trend provides a better model. In the VAP example, preexisting trends were assumed to be maintained for 3.5 years following the trials. While fairly constant trends in the control groups during this time period suggest that this assumption is reasonable, the longer the postintervention period the more questionable this premise becomes.

\section{Conclusions}

As the need for evidence-based, cost-effective care increases, there will be an increasing demand to assess the impact of new research and policy changes on cost of care and care delivery. Despite its limitations, ITSA represents a significant improvement over traditional observational study designs in quantifying the impact of an intervention. ITSA is a useful technique to understand, consider, and implement, especially as the field of neurosurgery evolves in sophistication in big-data analytics, economics, and health services research.

\section{References}

1. American Academy of Orthopaedic Surgeons: The Treatment of Symptomatic Osteoporotic Spinal Compression Fractures. Rosemont, IL: American Academy of Orthopaedic Surgeons, 2010

2. Abt NB, Bydon M, De la Garza-Ramos R, McGovern K, Olivi A, Huang J, et al: Concurrent neoadjuvant chemotherapy is an independent risk factor of stroke, all-cause morbidity, and mortality in patients undergoing brain tumor resection. J Clin Neurosci 21:1895-1900, 2014

3. Audit NP: Impact of NICE guidance on rates of haemorrhage after tonsillectomy: an evaluation of guidance issued during an ongoing national tonsillectomy audit. Qual Saf Health Care 17:264-268, 2008

4. Auerbach AD, Wachter RM, Cheng HQ, Maselli J, McDermott M, Vittinghoff E, et al: Comanagement of surgical patients between neurosurgeons and hospitalists. Arch Intern Med 170:2004-2010, 2010 
5. Buchbinder R, Osborne RH, Ebeling PR, Wark JD, Mitchell $\mathrm{P}$, Wriedt C, et al: A randomized trial of vertebroplasty for painful osteoporotic vertebral fractures. N Engl J Med 361:557-568, 2009

6. Cohen ME, Ko CY, Bilimoria KY, Zhou L, Huffman K, Wang X, et al: Optimizing ACS NSQIP modeling for evaluation of surgical quality and risk: patient risk adjustment, procedure mix adjustment, shrinkage adjustment, and surgical focus. J Am Coll Surg 217:336-346, 346.e1, 2013

7. Friedly JL, Comstock BA, Turner JA, Heagerty PJ, Deyo RA, Sullivan SD, et al: A randomized trial of epidural glucocorticoid injections for spinal stenosis. N Engl J Med 371:11-21, 2014

8. Glance LG, Osler TM, Mukamel DB, Meredith JW, Dick AW: Effectiveness of nonpublic report cards for reducing trauma mortality. JAMA Surg 149:137-143, 2014

9. Goz V, Errico TJ, Weinreb JH, Koehler SM, Hecht AC, Lafage V, et al: Vertebroplasty and kyphoplasty: national outcomes and trends in utilization from 2005 through 2010. Spine J 15:959-965, 2015

10. Hall BL, Hamilton BH, Richards K, Bilimoria KY, Cohen ME, Ko CY: Does surgical quality improve in the American College of Surgeons National Surgical Quality Improvement Program: an evaluation of all participating hospitals. Ann Surg 250:363-376, 2009

11. Heslin MJ, Taylor B, Hawn MT, Davies JE, Heslin RT, Mims $\mathrm{AH}$, et al: A $100 \%$ departmental mortality review improves observed-to-expected mortality ratios and University HealthSystem Consortium rankings. J Am Coll Surg 218:554-562, 2014

12. Imbens G, Rubin D: Causal Inference for Statistics, Social, and Biomedical Sciences: an Introduction. Cambridge, UK: Cambridge University Press, 2014

13. Kallmes DF, Comstock BA, Heagerty PJ, Turner JA, Wilson DJ, Diamond TH, et al: A randomized trial of vertebroplasty for osteoporotic spinal fractures. N Engl J Med 361:569579,2009

14. Maggard-Gibbons M: The use of report cards and outcome measurements to improve the safety of surgical care: the American College of Surgeons National Surgical Quality Improvement Program. BMJ Qual Saf 23:589-599, 2014

15. Manchikanti L, Pampati V, Hirsch JA: Analysis of utilization patterns of vertebroplasty and kyphoplasty in the Medicare population. J Neurointerv Surg 5:467-472, 2013

16. McCulloch P, Kreckler S, New S, Sheena Y, Handa A, Catchpole K: Effect of a "lean" intervention to improve safety processes and outcomes on a surgical emergency unit. BMJ 341:c5469, 2010
17. Mohr JP, Parides MK, Stapf C, Moquete E, Moy CS, Overbey JR, et al: Medical management with or without interventional therapy for unruptured brain arteriovenous malformations (ARUBA): a multicentre, non-blinded, randomised trial. Lancet 383:614-621, 2014

18. Morgan L, Pickering SP, Hadi M, Robertson E, New S, Griffin D, et al: A combined teamwork training and work standardisation intervention in operating theatres: controlled interrupted time series study. BMJ Qual Saf 24:111-119, 2015

19. Poulose BK, Ray WA, Arbogast PG, Needleman J, Buerhaus PI, Griffin MR, et al: Resident work hour limits and patient safety. Ann Surg 241:847-860, 2005

20. Rolston JD, Han SJ, Bloch O, Parsa AT: What clinical factors predict the incidence of deep venous thrombosis and pulmonary embolism in neurosurgical patients? J Neurosurg 121:908-918, 2014

21. Seicean A, Seicean S, Neuhauser D, Benzel EC, Weil RJ: 142 Race and outcomes after elective spine surgery. Neurosurgery 61 (Suppl 1):206, 2014

22. Smieliauskas F, Lam S, Howard DH: Impact of negative clinical trial results for vertebroplasty on vertebral augmentation procedure rates. J Am Coll Surg 219:525-533, 533.e1, 2014

23. Wagner AK, Soumerai SB, Zhang F, Ross-Degnan D: Segmented regression analysis of interrupted time series studies in medication use research. J Clin Pharm Ther 27:299-309, 2002

\section{Disclosures}

The authors report no conflicts of interest concerning the materials or methods used in this study or the findings specified in this paper.

\section{Author Contributions}

Conception and design: Lam. Acquisition of data: Smieliauskas. Analysis and interpretation of data: Smieliauskas. Drafting the article: Lam, Wong. Critically revising the article: all authors. Reviewed submitted version of manuscript: Lam. Statistical analysis: Smieliauskas. Administrative/technical/material support: Lam. Study supervision: Lam.

\section{Correspondence}

Sandi Lam, Department of Neurosurgery, Texas Children's Hospital, 6701 Fannin St., CCC Ste. 1230, Houston, TX 77030. email: sklam@texaschildrens.org. 